\title{
Research on Machine Translation of Deep Neural Network Learning Model Based on Ontology
}

Yaya Tian

School of Humanities, Shangluo University, Shangluo, Shaanxi, 726000, China

E-mail: Tianyaya19@outlook.com

Shaweta Khanna

JSS Academy of Technical Education, Noida, India

E-mail: shweta.khanna04@gmail.com

Anton Pljonkin

Southern federal university, Rastov-on-Don, Russia

E-mail: pljonkin@mail.ru

Keywords: ontology, deep neural network, machine translation, semantics, BLEU score, translation speed, crosslanguage information, translation skills

Received: May 21, 2021

To align different ontologies, it is necessary to find effective ways to achieve interoperability of information in the context of the Semantic Web. The development of accurate and reliable techniques to automatically perform this task, it is becoming more and more crucial as overlap between ontologies grows proportionally. In order to solve the problem that traditional machine translation cannot meet the needs of users because of the slow translation speed. According to the characteristics of Ontology's domain knowledge concept system, deep neural network learning model based machine translation method is proposed. Through the experimental design, we examine the translation time and BLEU score and other indicators. After junior translators use the tools, the translation time is reduced by $34.0 \%$ and the BLEU score increases by 7.59; after the senior translators use the tools, the translation time is reduced by $11.3 \%$, and the BLEU score is increased by 1.67. Analysis of the experimental results shows that the essence of this method is to complement translation skills, so it is more effective for junior translators who are not good enough in translation skills. The machine translation method based on deep neural network learning can significantly improve the quality and efficiency of translation.

Povzetek: Predstavljena je metoda prevajanja s pomočjo globokih nevronskih mrež in ontologij.

\section{Introduction}

The language-neutral world and linguistic knowledge are fully integrated by the machine translation system which is of very high quality. The world knowledge is widely modeled by utilizing the ontology at the conceptual level. This article introduces a deep neural network learning model machine translation system based on ontology. In this system, ontology is used as a model of world knowledge [1]. It is organized by organizing concepts. One of the main application areas of machine translation is the translation of scientific documents. [2-4]. According to the sequence of translation methods, machine translation methods can be further divided into statistical machine translation methods, and deeplearning based translation methods.

For the many applications like computer vision, speech recognition, natural language processing, multitask, the high performance is achieved by the deep learning [5]. It is also known as representation learning in which aspect of automatic representation learning is highlighted. For characterizing biological phenomena, main features biomedical ontologies provide are controlled vocabularies. The phenotypes are observed in model organism databases are characterized by the

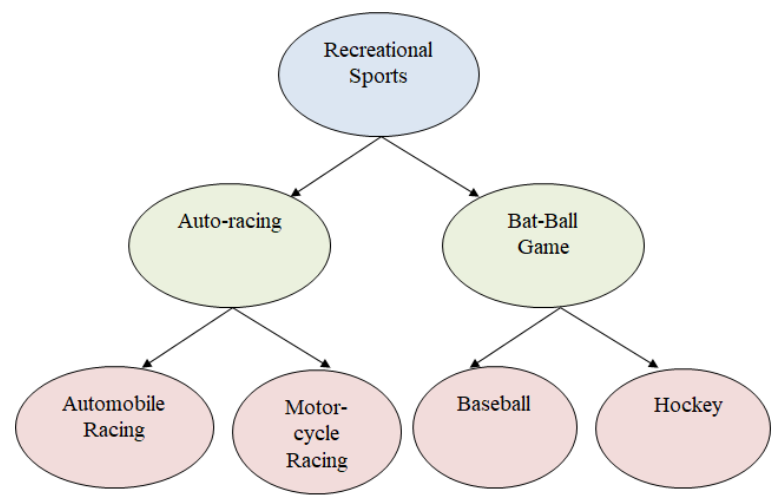

Figure 1: A sample ontology for sports domain. 
phenotype ontologies [9-12]. The concepts, relationships, axiomatic constraints and the domain knowledge's formal representation are provided by ontology. The sample of ontology is shown in Figure 1. The regularities from data with various semantic granularities are encoded by the set of representations [13-17].

The core idea is to use the distributed representation of deep learning methods to solve the problem of statistical translation methods for modeling the global context and deep semantic information [18-20]. Traditional statistical machine translation has two problems: One is its complicated translation model, and the other is its poor handling of long-distance dependencies. However, machine translation based on deep learning has been able to deal with the problem of long-distance dependence well due to the introduction of sequence models and the recently proposed attention mechanism [21]. At the same time, this end-to-end translation model greatly simplifies the traditional statistical machine translation model. Therefore, machine translation based on deep learning has been developed rapidly in recent years, which has attracted widespread attention from industry and academia. The rest of the paper is organized as follows. Section II provides an overview of the exhaustive literature survey along with the contribution of the paper followed by a methodology adopted in section III. The experimental design is explained in section IV. A detailed discussion of obtained results is in section V. Finally, concluding remarks are provided in section VI.

\section{Literature review}

Using semantic web technology to deal with homonyms and homonyms semantically and others based on ontology of Arabic English machine translation model NAN, this model can simulate human translation. [22]. Firstly, the multi-layer $\mathrm{NN}$ is combined with the undirected probability model to utilize the similarity and context information efficiently to model word alignment. Secondly, low size will be used. The feature representation is combined with other features into the preorder model of linear ranking Xia, Y. to construct the preorder model named NNPR. Combining the word alignment model and preorder model in the same deep neural network, this is a deep neural networks based on the statistical machine translation model.

In major biological database, ontologies have long been engaged in the life sciences. In the machine learning models, the background knowledge in similarity-based analysis is provided by the ontologies [23]. There is a great development of the methods employed the combination of ontologies and machine learning. Author in this paper provides the overview of ontologies methods for the similarity computation. The background knowledge in ontologies is exploited by the ontology embedding which measures the semantic similarity. [24]. A translator-based operational interoperability model is defined for cyber-physical systems interaction. The translator learning task and machine learning tasks are presented by the author. [25].
The deep neural networks are trained by employing the state-of-the-art methods. The several experiments are performed and presented for accurately performing the ontology reasoning on very large and challenging benchmarks. There are different obstacles in which logicbased symbolic reasoning are prohibited [26]. By introducing formal semantics into deep learning process, a semantic rich deep learning model is designed from a knowledge driven perspective. Author proposed ontology-based deep restricted Boltzmann machine (OBDRBM) which utilized for designing the deep restricted Boltzmann machines (DRBM) architecture [27]. The amount of data that needs to be represented grows proportionally as the semantic web technologies are expanding and becoming more popular. Among knowledge bases, redundancy occurs since web technologies are designed to be decentralized. The machine learning techniques based approach is presented by the author and the semantic information can be retrieved automatically from text information within ontologies. The convolution neural networks were utilized for this work. [28]. It is shown that the deep learning methods result in high performance in the field of vision, speech recognition, machine translation, art, medical imaging etc. The Convolutional Neural Network $(\mathrm{CNN})$, Recurrent Neural Network (RNN), including Long Short-Term Memory (LSTM) and Gated Recurrent Units (GRU), are covered in this survey.

This paper designs a machine online translation system based on the deep neural network method. According to the characteristics of Ontology's domain knowledge concept system, a machine translation method based on deep neural network learning model is proposed. It is a major innovation of cross-language information retrieval technology in my country and has a wide range of social and industrial application values.

\section{Research methods}

\subsection{Neural network machine translation model}

The neural network machine translation model can be directly mapped to the output sequence in an end-to-end manner. If the given source language is:

$$
X=\left\{x_{1}, x_{2}, \ldots x_{n}\right\}
$$

Set the target language as:

$$
Y=\left\{y_{1}, y_{2}, \ldots, y_{n}\right\}
$$

Then the modeling translation probability based on neural network is expressed as:

$$
p(Y \mid X)=\prod_{t=1}^{m} P\left(y_{t} \mid y<t, X\right)
$$

In the formula, $y<t=y_{1}, y_{2}, \ldots, y_{t-1}$, apply GRU, LSTM and other non-linear units to the neural network machine translation model, with the current input $x_{t}$ hidden state. $h_{t-1}$, calculate the hidden state $h_{t}$ :

$$
h_{t}=R N N\left(h_{t-1}, x_{t}\right)
$$


In order to effectively enrich the context information, through the source language of the encoding segment, the encoding results in different directions.

Integration, making it the final hidden state, this process is expressed as:

$$
\begin{array}{r}
\overrightarrow{\boldsymbol{h}_{t}}=\operatorname{RNN}\left(\overrightarrow{\boldsymbol{h}}_{t-1}, x_{t}\right) \\
\overrightarrow{\boldsymbol{h}_{t}}=\operatorname{RNN}\left(\overleftarrow{\boldsymbol{h}}_{t-1}, x_{t}\right) \\
\boldsymbol{h}_{t}=\left[\begin{array}{l}
\overrightarrow{\boldsymbol{h}}_{t} \\
\overleftarrow{\boldsymbol{h}}_{t}
\end{array}\right]
\end{array}
$$

Realize the generation of the target language through the decoder side:

$$
\begin{gathered}
q=g\left(y_{t-1}, c_{t}, s_{t}\right) \\
p\left(y_{t} \mid y<t, X\right)=\operatorname{soft} \max (q)
\end{gathered}
$$

The $q$ in the formula refers to the target end word tensor that needs to be predicted, $g($.$) refers to the$ nonlinear element, $s_{t}$ refers to the hidden state on the decoder side. The $c_{t}$ refers to the weighted sum calculation of all hidden states at the source $c_{t}$.

The method is:

$$
\begin{gathered}
c_{t}=\operatorname{artention}\left(s_{t-1}, h\right)=\sum_{i=1}^{n} a_{t},{ }_{i} h_{i} \\
a_{t, i}=\operatorname{softmax}\left(V_{a}^{T} \operatorname{tamh}\left(W_{a} s_{t-1}+U_{a} h_{j}\right)\right)
\end{gathered}
$$

In the formula $V_{a}, W_{a}, U_{a}$ refers to neural network training parameters. Figure 2 shows the structure of neural network machine translation.

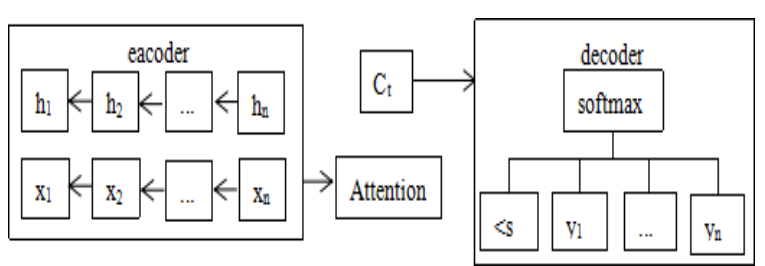

Figure 2: The structure of neural network machine translation.

\subsection{Design of machine online translation system}

\subsubsection{The overall structure of the system}

The machine online translation system designed in this paper mainly includes knowledge management, translation subsystem, and user interface [29, 30]. Figure 3 shows the overall system structure. The rule-based method of the system model is based on the rule method and analyzed through the source language. It logically includes three stages of generation, conversion and analysis. This method uses knowledge sources as a rule base and a comprehensive dictionary. Based on the deep neural network model through the knowledge base, the case library is expanded to become the case pattern library. To put it simply, the similar structure instances in the library are abstracted into sentence patterns with variables and stored in the library. When translating, the input sentence is matched with the source language part of the pattern in the library. In the process of sentence analysis, the embedded person is based on the method of case pattern. The system realizes the effective output of translation results based on the influence model method. When the example pattern library lacks influence patterns and sentences similar to the input source language, the system inputs the translation result.

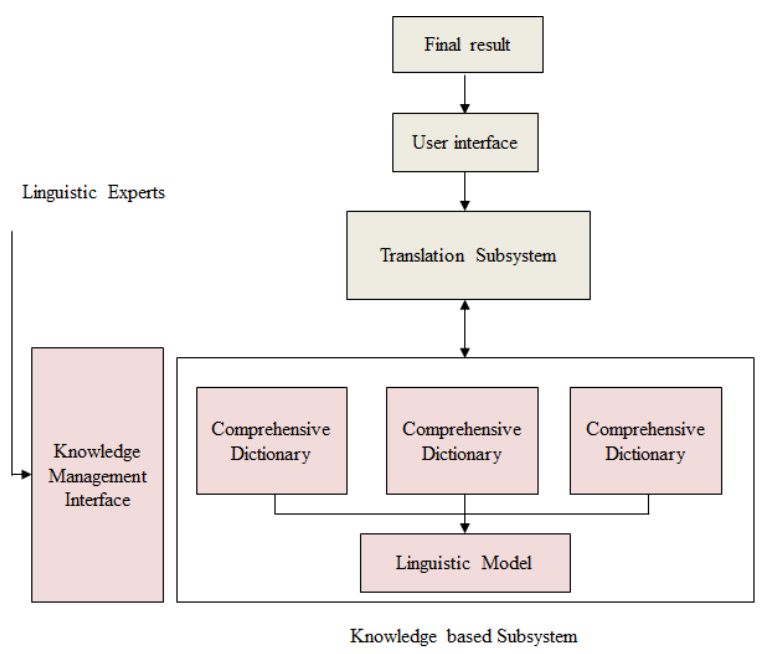

Figure 3: The overall structure of the system.

\subsubsection{Design of translation subsystem}

In the system design process, there are 8 interconnected functional modules in the translation subsystem. Figure 4 shows the system translation process structure. Modules such as part-of-speech tagging, lexical analysis and shallow syntax can analyze the source language, and the realization of instance module matching takes the instance pattern as the basis [31]. The phrase target generation module is the main destination, which is the output of the target translation.

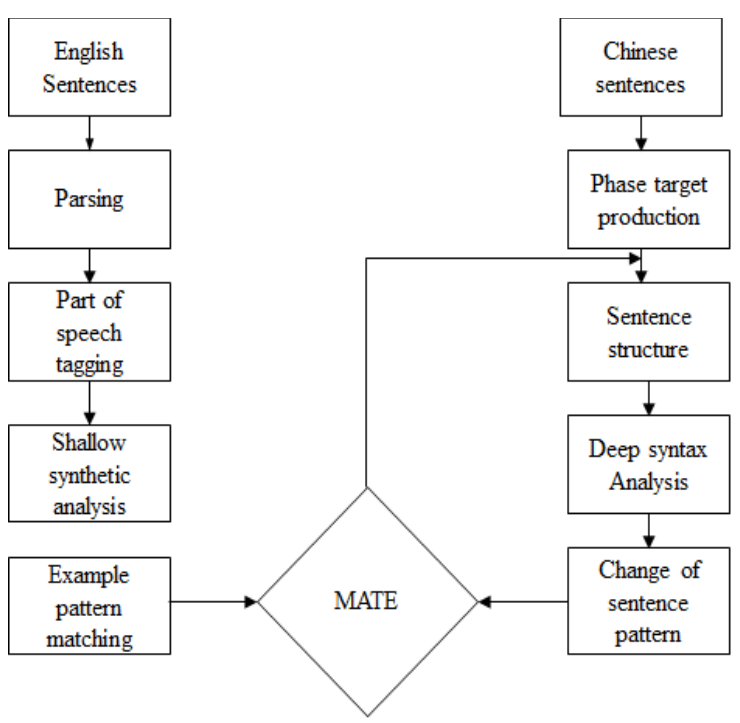

Figure 4: System translation process structure. 


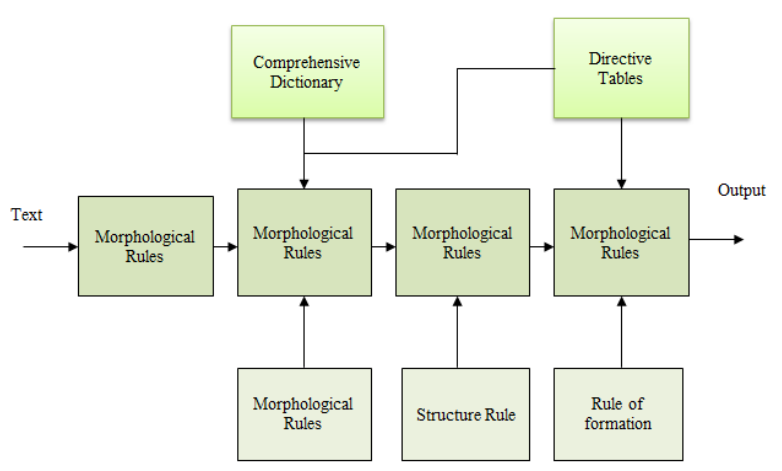

Figure 5: The structural model of the lexical analyzer.

The knowledge source of morphological translation is the knowledge of linguistic rules, which is also the process of transforming real language text into part-ofspeech sequence. The system syntax analyzer includes uncollected, preprocessed word processing, and analyzes the self-learning results. The structural model of the lexical analyzer is shown in Figure 5.

Part-of-speech tagging refers to the automatic tagging of the words of the input sentence in the context. The system uses the method of combining rules and statistics to mark the part of speech [32]. Sentence pattern transformation is the transitional stage of translation based on transformation generation. First, the sentence type is judged by sentence punctuation and structure [33-35]. If it is an interrogative sentence, it is transformed into a Chen data structure for different types; if it is a declarative sentence, the sentence information should be recorded; if it is an imperative sentence or an exclamation sentence, the translation is implemented based on the matching of the example pattern.

\subsubsection{Web service of machine translation}

The machine translation Web server specification is a logical relationship, which is added to the J2EE platform, mainly including the port components and SOAP transmission provided by the Web container and the EJB container. J2EE Web services must be mapped to existing J2EE platform roles through a single port [3638]. For example, J2EE Web service product provider roles are mapped to J2EE product provider roles.

The Web server client includes another Web service, J2EE component, or any Java application. The Web service client view can realize remote, which provides local and remote transparency [39, 40]. The online translation system can provide another Web server, filter and disable Web service requests, and provide security constraints and login.

\section{Experimental designs}

\subsection{Realization of machine online translation system}

In order to verify the effectiveness of the system, an English-Chinese translation experiment is carried out to analyze the role and influence of the intelligent translation system in the translator's translation process. The experiment is divided into four groups, as shown in Table 1. A preliminary test was conducted for juniors in the English Translation Department, and 20 students with equivalent translation levels were selected as Group A (as junior translators); A preliminary test was conducted on employees of translation companies with more than 5 years of translation experience, and the selection of equivalent translation levels of 20 employees are regarded as group B (as professional translators).

\begin{tabular}{|c|c|c|c|c|}
\hline $\begin{array}{c}\text { Experiment } \\
\text { grouping }\end{array}$ & $\begin{array}{c}\text { Translator } \\
\text { category }\end{array}$ & $\begin{array}{c}\text { Whether } \\
\text { to use } \\
\text { tools }\end{array}$ & $\begin{array}{c}\text { Test } \\
\text { document }\end{array}$ & Test index \\
\hline $\begin{array}{c}\text { Group A1- } \\
\text { A10 }\end{array}$ & $\begin{array}{l}\text { Junior } \\
\text { translator }\end{array}$ & Yes & One with & Translation \\
\hline $\begin{array}{c}\text { A11- A20 } \\
\text { group }\end{array}$ & $\begin{array}{l}\text { Junior } \\
\text { translator }\end{array}$ & no & $\begin{array}{c}\text { There are } \\
578\end{array}$ & BLUE \\
\hline $\begin{array}{c}\text { Group B1- } \\
\text { B10 }\end{array}$ & $\begin{array}{l}\text { Senior } \\
\text { translator }\end{array}$ & Yes & $\begin{array}{c}\text { The English } \\
\text { of the } \\
\text { word }\end{array}$ & $\begin{array}{l}\text { Translation } \\
\text { efficiency: }\end{array}$ \\
\hline $\begin{array}{l}\text { Group } \\
\text { B11-B20 }\end{array}$ & $\begin{array}{l}\text { Senior } \\
\text { translator }\end{array}$ & no & Document & $\begin{array}{c}\text { Translation } \\
\text { time }\end{array}$ \\
\hline
\end{tabular}

Table 1: Experimental design.

\begin{tabular}{ccc}
\hline $\begin{array}{c}\text { Translator } \\
\text { Number }\end{array}$ & $\begin{array}{c}\text { Translation } \\
\text { time }\end{array}$ & $\begin{array}{c}\text { BLEU } \\
\text { score }\end{array}$ \\
\hline A-1 & $1: 02: 30$ & 88.40 \\
A-2 & $0: 50: 30$ & 77.90 \\
A-3 & $0: 30: 09$ & 67.70 \\
A-4 & $0: 54: 32$ & 76.90 \\
A-5 & $0: 52: 40$ & 71.40 \\
\hline
\end{tabular}

Table 2: Experimental results of tools used by junior translators.

\begin{tabular}{ccc}
\hline $\begin{array}{c}\text { Translator } \\
\text { Number }\end{array}$ & $\begin{array}{c}\text { Translation } \\
\text { time }\end{array}$ & $\begin{array}{c}\text { BLEU } \\
\text { score }\end{array}$ \\
\hline A-11 & $0: 59: 15$ & 50.05 \\
A-12 & $1: 20: 10$ & 74.00 \\
A-13 & $0: 49: 05$ & 69.20 \\
A-14 & $1: 05: 53$ & 70.90 \\
A-15 & $1: 30: 26$ & 72.90 \\
\hline
\end{tabular}

Table 3: Experimental results of junior translators not using tools.

\section{Experimental results and discussion}

The experimental results are shown in Table 2 to Table 5. The graphical representation of senior translators without tools is presented in Figure 6. Calculate the average 
number of translation time and BLEU score for each group, summarized in Table 6 and Figure 7 shows the graphical representation for better analysis.

\begin{tabular}{ccc}
\hline $\begin{array}{c}\text { Translator } \\
\text { Number }\end{array}$ & $\begin{array}{c}\text { Translation } \\
\text { time }\end{array}$ & $\begin{array}{c}\text { BLEU } \\
\text { score }\end{array}$ \\
\hline B-1 & $1: 02: 43$ & 85.60 \\
B-2 & $0: 52: 40$ & 86.90 \\
B-3 & $0: 49: 18$ & 75.20 \\
B-4 & $0: 55: 25$ & 77.80 \\
B-5 & $1: 00: 45$ & 86.00 \\
\hline
\end{tabular}

Table 4: Experimental results of tools used by senior translators.

\begin{tabular}{ccc}
$\begin{array}{l}\text { Translator } \\
\text { Number }\end{array}$ & $\begin{array}{c}\text { Translation } \\
\text { time }\end{array}$ & $\begin{array}{c}\text { BLEU } \\
\text { score }\end{array}$ \\
\hline B-11 & $1: 14: 30$ & 79.50 \\
B-12 & $0: 55: 10$ & 81.10 \\
B-13 & $1: 20: 10$ & 89.20 \\
B-14 & $0: 58: 26$ & 72.20 \\
B-15 & $0: 50: 30$ & 82.20 \\
\hline
\end{tabular}

Table 5: Experimental results of senior translators without tools.

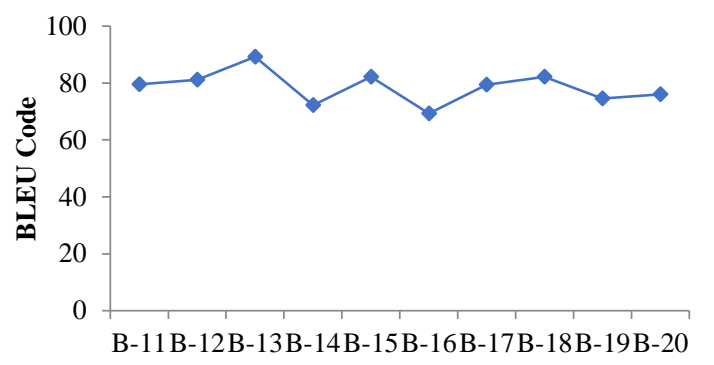

Translator code $\mathbf{C}$

Figure 6: Experimental results of senior translators without tools.

\begin{tabular}{cccc}
\hline & $\begin{array}{c}\text { Experiment } \\
\text { grouping }\end{array}$ & $\begin{array}{c}\text { Transl } \\
\text { ation time }\end{array}$ & $\begin{array}{c}\text { BLE } \\
\text { U score }\end{array}$ \\
\hline \multirow{2}{*}{$\begin{array}{ccc}74.7 \\
\text { A }\end{array}$} & use tools & $0: 49: 57$ & 7 \\
& No tools & $1: 15: 18$ & 67.1 \\
& & & 8 \\
Group & use tools & $0: 55: 15$ & 80.2 \\
B & & & 2 \\
& No tools & $1: 01: 50$ & 78.5 \\
& & & 5 \\
\hline
\end{tabular}

Table 6: Data analysis.
The use of tools in the experiment of Group A reduced the translation time by $34.0 \%$ compared with no

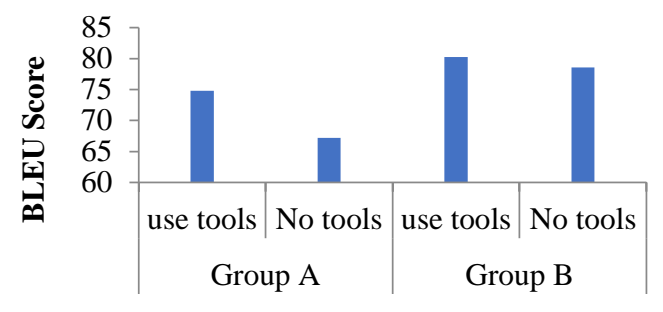

Experiment Grouping

Figure 7: Data analysis.

tools, and the BLEU score increased by 7.59. As compared with no tools, the translation time of group B experiment with tools reduced by $11.3 \%$, and the BLEU score increased by 1.67. The improvement in translation efficiency and quality of group A was significantly greater than that of group B. The reason is that the essence of this method is to complement translation skills, so it is less effective for senior translators who have already excellent translation skills.

The above experiments also verify the correctness of the research objectives of this article. This method can improve translation productivity and enable junior translators to reach the level of higher-level translators with the help of translation tools.

\section{Conclusion}

This paper makes a preliminary analysis of the machine translation technology learned by deep neural networks, and realizes the creation of a prototype system through research ideas. Based on cases and experiments, the feasibility of technical ideas are verified, the use of this technology in translation can innovate my country's cross-language information retrieval technology, and has a wide range of industrial application value and social value. In recent years, artificial intelligence technology has flourished. Significant progress has been made in the fields of image, voice or video. Although natural language processing is called the jewel in the crown of artificial intelligence, it is still difficult to understand and process natural language, and the effect of the representative application of machine translation is still not very satisfactory. The emergence of deep learning undoubtedly opened a breakthrough for machine translation. At present, the deep neural network learning based on the autonomous training of the system has been manually adjusted. After junior translators use the tools, the translation time is reduced by $34.0 \%$ and the BLEU score increases by 7.59 ; after the senior translators use the tools, the translation time is reduced by $11.3 \%$, and the BLEU score is increased by 1.67. Analysis of the experimental results shows that the essence of this method is to complement translation skills, so it is more effective for junior translators who are not good enough in translation skills. In the future, automatic learning will 
be realized through the establishment of statistical algorithms and models.

\section{Acknowledgement}

Research Center of Shangluo Culture and Jia Pingwa, A Study on the Translation and Introduction of Mo Yan's Works and Jia Pingwa's Works in English-speaking Countries (18SLWH05).

\section{References}

[1] Ren, B., Li, J., Zheng, Y., Chen, X., Zhao, Y., \& Zhang, H., et al. (2020). Research on fault location of process-level communication networks in smart substation based on deep neural networks. IEEE Access, 8, 109707-109718.

DOI: https://doi.org/10.1109/ACCESS.2020.30021 72

[2] Zappone, A., Di Renzo, M., \& Debbah, Mérouane. (2019). Wireless networks design in the era of deep learning: model-based, ai-based, or both?. IEEE Transactions on Communications, 67(10), 73317376.

DOI: https://doi.org/10.1109/TCOMM.2019.29240 10

[3] Moussallem, D., Wauer, M., \& Ngomo, A. C. N.. (2018). Machine translation using semantic web technologies: a survey. Journal of Web Semantics, 51(AUG.), 1-19. DOI : https://doi.org/10.1016/j.websem.2018.07.001

[4] Dahan, N. A., \& Ba-Alwi, F. M.. (2019). Extending a model for ontology-based arabic-english machine translation. International Journal of Artificial Intelligence \& Applications, 10(01), 55-67. DOI : https://doi.org/10.5121/ijaia.2019.10105 55

[5] Xia, Y.. (2020). Research on statistical machine translation model based on deep neural network. Computing, 102(3), 643-661.

DOI: https://doi.org/10.1007/s00607-019-00752-1

[6] Petrucci, G., Rospocher, M., \& Ghidini, C.. (2018). Expressive ontology learning as neural machine translation. Journal of Web Semantics, 52(OCT.), 66-82.

DOI: https://doi.org/10.1016/j.websem.2018.10.002

[7] Astrakhantsev, N. (2018). Atr4s: toolkit with stateof-the-art automatic terms recognition methods in scala. Language Resources \& Evaluation, 52(3), 853-872. https://doi.org/10.1007/s10579-017-9409-4

[8] Ma, S. Zhang, Y., \& Zhang, C.. (2018). Using multiple web resources and inference rules to classify chinese word semantic relation. Information Discovery and Delivery, 46(2), 00-00. DOI: https://doi.org/10.1162/tacl_a_00316

[9] Bei, L.. (2020). Study on the intelligent selection model of fuzzy semantic optimal solution in the process of translation using english corpus. Wireless Communications and Mobile Computing, 2020(5), 1-7. https://doi.org/10.1155/2020/8827657
[10] Sharma, A., \& Kumar, R. (2019). Service-level agreement - energy cooperative quickest ambulance routing for critical healthcare services. Arabian Journal for Science and Engineering, 44(4), 3831-3848.

https://doi.org/10.2174/22132759116661810251252 24

[11] Utomo, F. S., Suryana, N., \& Azmi, M. S.. (2020). Stemming impact analysis on indonesian quran translation and their tafsir classification for ontology instances. IIUM Engineering Journal, 21(1), 33-50.

https://doi.org/10.31436/iiumej.v21i1.1170purata

[12] Chen, Y., Sabri, S., Rajabifard, A., \& Agunbiade, M. E.. (2018). An ontology-based spatial data harmonisation for urban analytics. Computers, Environment and Urban Systems, 72(NOV.), 177190.https://doi.org/10.1016/j.compenvurbsys.2018. 06.009

[13] Zheng, T., Gao, Y., Wang, F., Fan, C., Fu, X., Li, M., \& Ma, H. (2019). Detection of medical text semantic similarity based on convolutional neural network. BMC medical informatics and decision making, 19(1), 1-11. https://doi.org/10.3390/info11050241

[14] Sharma, A., \& Kumar, R. (2019). Risk-energy aware service level agreement assessment for computing quickest path in computer networks. International Journal of Reliability and Safety, 13(1-2), 96-124. https://doi.org/10.1155/2019/4109453

[15] García-Díaz, J. A., Cánovas-García, M., \& Valencia-García, R. (2020). Ontology-driven aspect-based sentiment analysis classification: An infodemiological case study regarding infectious diseases in Latin America. Future Generation Computer Systems, 112, 641-657.

DOI: https://doi.org/10.1016/j.future.2020.06.019

[16] Petrucci, G., Rospocher, M., \& Ghidini, C. (2018). Expressive ontology learning as neural machine translation. Journal of Web Semantics, 52, 66-82. DOI: https://doi.org/10.1016/j.websem.2018.10.002

[17] Xiao, Y., Xing, C., Zhang, T., \& Zhao, Z. (2019). An intrusion detection model based on feature reduction and convolutional neural networks. IEEE Access, 7, 42210-42219. https://doi.org/10.3390/s21020626

[18] Sharma, A., \& Kumar, R. (2019). Computation of the reliable and quickest data path for healthcare services by using service-level agreements and energy constraints. Arabian Journal for Science and Engineering, 44(11), 9087-9104. https://doi.org/10.1007/s13369-019-03836-4

[19] Chen, R., \& Sharma, A. (2021). Construction of complex environmental art design system based on $3 \mathrm{D}$ virtual simulation technology. International Journal of System Assurance Engineering and Management, 1-8. DOI: https://doi.org/10.1007/s13198-021-01104-z

[20] Hung, C., \& Wermter, S. (2004). Neural network based document clustering using wordnet 
ontologies. International Journal of Hybrid Intelligent Systems, 1(3-4), 127-142. DOI: https://doi.org/10.3233/HIS-2004-13-402

[21] Zhang, W., Li, R., Zeng, T., Sun, Q., Kumar, S., Ye, J., \& Ji, S. (2016). Deep model based transfer and multi-task learning for biological image analysis. IEEE transactions on Big Data, 6(2), 322333. https://doi.org/10.1007/s11263-014-0767-8

[22] Ren, X., Li, C., Ma, X., Chen, F., Wang, H., Sharma, A., Gaba, G.S. and Masud, M., 2021. Design of multi-information fusion based intelligent electrical fire detection system for green buildings. Sustainability, 13(6), p.3405. DOI: https://doi.org/10.3390/su13063405

[23] Dhaini, Mahdi, Mohammad Jaber, Amin Fakhereldine, Sleiman Hamdan, and Ramzi A. Haraty. "Green Computing Approaches-A Survey." Informatica 45, no. 1 (2021). https://doi.org/10.31449/inf.v45i1.2998

[24] Zhou, Y., Huang, C., Hu, Q., Zhu, J., \& Tang, Y. (2018). Personalized learning full-path recommendation model based on LSTM neural networks. Information Sciences, 444, 135-152. doi: https://doi.org/10.1016/j.ins.2018.02.053

[25] Zhang, C., \& Zhou, G. (2019). A view-based 3D CAD model reuse framework enabling product lifecycle reuse. Advances in Engineering Software, 127, 82-89. DOI: https://doi.org/10.1016/j.advengsoft.2018.09.001.

[26] Mureşan, H., \& Oltean, M. (2018). Fruit recognition from images using deep learning. Acta Universitatis Sapientiae, Informatica, 10(1), 26-42, DOI: https://doi.org/10.2478/ausi-2018-0002.

[27] Kulmanov, M., Smaili, F. Z., Gao, X., \& Hoehndorf, R. (2020). Semantic similarity and machine learning with ontologies. https://doi.org/10.1093/bib/bbaa199

[28] Nilsson, J., Sandin, F., \& Delsing, J. (2019, July). Interoperability and machine-to-machine translation model with mappings to machine learning tasks. In 2019 IEEE 17th International Conference on Industrial Informatics (INDIN) (Vol. 1, pp. 284289). IEEE. doi: https://doi.org/10.3390/s21041309

[29] Hohenecker, P., \& Lukasiewicz, T. (2020). Ontology reasoning with deep neural networks. Journal of Artificial Intelligence Research, 68, 503540. DOI : https://doi.org/10.1613/jair.1.11661

[30] Wang, H., Dou, D., \& Lowd, D. (2016, September). Ontology-based deep restricted boltzmann machine. In International Conference on Database and Expert Systems Applications (pp. 431-445). Springer, Cham. https://doi.org/10.1016/j.procs.2019.09.198

[31] Bento, A., Zouaq, A., \& Gagnon, M. (2020, May). Ontology matching using convolutional neural networks. In Proceedings of The 12th Language Resources and Evaluation Conference (pp. 56485653). doi: https://doi.org/10.3390/app10217909

[32] Alom, M. Z., Taha, T. M., Yakopcic, C., Westberg, S., Sidike, P., Nasrin, M. S., ... \& Asari, V. K. (2019). A state-of-the-art survey on deep learning theory and architectures. Electronics, 8(3), 292. https://doi.org/10.3390/electronics 8030292

[33] Hung, C., \& Wermter, S. (2004). Neural network based document clustering using wordnet ontologies. International Journal of Hybrid Intelligent Systems, 1(3-4), 127-142.

DOI: https://doi.org/10.3233/HIS-2004-13-402

[34] Chung, K., Yoo, H., \& Choe, D. E. (2020). Ambient context-based modeling for health risk assessment using deep neural network. Journal of Ambient Intelligence and Humanized Computing, 11(4), 1387-1395.

DOI: https://doi.org/10.1007/S12652-018-1033-7

[35] Utkin, L. V., \& Zhuk, K. D. (2020). Improvement of the deep forest classifier by a set of neural networks. Informatica, 44(1), DOI: https://doi.org/10.31449/inf.v44i1.2740

[36] hou, Q., Yan, P., Liu, H., \& Xin, Y. (2019). A hybrid fault diagnosis method for mechanical components based on ontology and signal analysis. Journal of Intelligent Manufacturing, 30(4), 16931715. https://doi.org/10.1007/s10845-017-1351-1

Sharma, A., Kumar, R., \& Bajaj, R. K. (2021). On Energy-constrained Quickest Path Problem in Green Communication Using Intuitionistic Trapezoidal Fuzzy Numbers. Recent Advances in Computer Science and Communications (Formerly: Recent Patents on Computer Science), 14(1), 192200, DOI: https://doi.org/10.2174/22132759116661 81025125224

[37] Morkūnas, M., Treigys, P., Bernatavičienè, J., Laurinavičius, A., \& Korvel, G. (2018). Machine learning based classification of colorectal cancer tumour tissue in whole-slide images. Informatica, 29(1), 75-90,

DOI:https://doi.org/10.15388/Informatica.2018.158

[38] Poongodi, M., Sharma, A., Vijayakumar, V., Bhardwaj, V., Sharma, A. P., Iqbal, R., \& Kumar, R. (2020). Prediction of the price of Ethereum blockchain cryptocurrency in an industrial finance system. Computers \& Electrical Engineering, 81, 106527.

https://doi.org/10.1016/j.compeleceng.2019.106527

[39] Zhang, X., Wang, R., Sharma, A., \& Gopal, G. (2021). Artificial intelligence in cognitive psychology-Influence of literature based on artificial intelligence on children's mental disorders. Aggression and Violent Behavior, 101590.

DOI: https://doi.org/10.1016/j.avb.2021.101590 
